\title{
Empirische Studie zur Erlangung der interkulturellen Kompetenz deutscher Führungskräfte zur Vorbereitung auf die Entsendung in die VR China
}

\author{
Stephanie Nagel
}

\section{Vorbemerkung}

Im Rahmen der sich erweiternden Beziehungen zwischen dem Reich der Mitte und der Bundesrepublik Deutschland ist die Frage der interkulturellen Kompetenz deutscher Fachleute, die in China tätig sind, von immer größerer Bedeutung geworden. Die nachstehende Arbeit versucht, die Vorbereitungsmaßnahmen der Unternehmen gegenuiber ihren Angestellten zu analysieren und zu evaluieren. Dabei sind zum Teil sehr ernuichternde Ergebnisse zu verzeichnen.

Auch die Erfahrungen des Autors, der seit 22 Jahren in und mit China tätig ist, zeigen, dass die Vorbereitung auf einen Tätigkeitsaufenthalt in China erheblich informativer sein sollte. Noch immer werden viele Entscheidungsträger aus der Industrie ohne interkulturelle und ohne Sprachvorbereitung nach China entsandt.

Die Mitwirkung des Autors bei der Inbetriebnahme von Metro Shanghai hatte eindeutig nachgewiesen, dass Sprachkompetenz verbunden mit Landes- und fachspezifischen Kenntnissen zu einer Bevorzugung führt gegenüber den nur in der Fachkompetenz besseren Mitarbeitern. Als Beispiel soll folgender Sachverhalt genannt werden: In der Endphase der Inbetriebnahmevorbereitungen des ersten Teilstücks der Linie eins der Metro Shanghai (SMC) hatte sich kurzfristig der Bürgermeister von Shanghai zu einer Probefahrt entschlossen. Der erste Zug war von AEG noch nicht übergeben und die Strecke nur bedingt betriebsfähig. In diesem Dilemma suchte nun der General Manager der SMC die Unterstuitzung des Autors, der aber bei weitem nicht so bahnfachkompetent war wie die anderen Mitarbeiter aus der Bundesrepublik. Der entscheidende Grund für die Wahl des Autors war - wie der General Manager später verriet die Möglichkeit, direkt zu kommunizieren und dabei eine bestimmte Grundsachkompetenz zu nutzen.

Dieses Beispiel soll verdeutlichen, dass ohne eine solide Weiterbildung im interkulturelle Kompetenz ein Erfolg der deutschen Unternehmen in China auf die Dauer eher unbefriedigend ist. Die Autoren hoffen, mit diesem Artikel einen Beitrag zum „Wachruitteln“ der China orientierten Unternehmen geleistet zu haben.

Dr. Dirk Forschner, China-Institut

\section{Einführung in das Thema der Diplomarbeit}

Mit der Einschreibung im September 1998 an der TFH Wildau im Studiengang der Betriebswirtschaftslehre mit dem Schwerpunkt Internationales Marketing nahm die Autorin die Gelegenheit war, am dortigen China-Institut bei Dr. Dirk Forschner die chinesische Sprache zu erlernen und einen einmonatigen Sommersprachkurs an der International Youth University in Beijing zu absolvieren. Im direkten Anschluss daran folgte ein siebenmonatiges Praktikum bei Dräger Medical Instrument in Shanghai. Während dieses Praktikums ergab sich die Möglichkeit, ausländische Führungskräfte kennen zu lernen, die nur wenig über die chinesische Kultur und deren Mentalität wussten. Aufgrund dessen ergab sich die Frage, ob diese Situation auch fuir deutsche Führungskräfte zutreffend sein würde. Daher war die alles entscheidende Fragestellung für die zu erarbeitende Diplomarbeit, ob und inwiefern deutsche Führungskräfte fuir einen Chinaaufenthalt vorbereitet werden, da aufgrund der Erfahrungen im Praktikum die Annahme bestand, dass deutsche Führungskräfte nicht ausreichend interkulturell vorbereitet und versiert sind.

\section{Zielsetzung der Arbeit}

Damit die deutsche Wirtschaft an dem dynamischen chinesischen Markt partizipieren kann, müssen Präventionsmaßnahmen durchgefuihrt werden, um den kulturspezifischen Herausforderungen des chinesischen Marktes gewachsen zu sein. Ein erfolgreiches Engagement in China kann daher erst sichergestellt werden, wenn die zu entsendenden Führungskräfte zuvor gezielt vorbereitet und qualifiziert wurden. Sie sollten die erforderlichen Informationen erhalten und auf die Andersartigkeit der Führungsqualitäten vorbereitet sein.

Dementsprechend wurde für den empirischen Teil der Arbeit ein Fragebogen entwickelt, um die erwähnte Annahme feststellen zu können. Dieser half, die vorhandenen Informationen und Kenntnisse der Führungskräfte über China zu bewerten und darüber hinaus den Informations- und Bildungsbedarf zu ermitteln, der für eine Führungskraft in der VR China unbedingt erforderlich ist.

Daher ist das zentrale Anliegen der empirischen Untersuchung die Ermittlung des Status quo entsprechender Vorbereitungsmaßnahmen für Führungskräfte, und, unter Mitwirkung dieser, deren Verbesserung. 


\section{Probanden und Auswahlumfang}

Die empirische Studie stuitzt sich auf die Expertenbefragung von Unternehmen, die bereits seit geraumer Zeit in China aktiv sind. Damit konnte gewährleistet werden, dass die Experten über einen entsprechenden Erfahrungshorizont verfuigen. Aufgrund dessen umfasste die Studie nur solche deutsche Unternehmen, die entweder Repräsentanzen, Dienstleistungsbüros oder Jointventures und deutsche Mitarbeiter in der VR China haben. Die Erhebung der geeigneten Unternehmen wurde durch eine weniger konzentrierte Befragung durchgefuihrt, um eine höhere Erreichbarkeit dieser zu erzielen. Darüber hinaus entschloss sich die Autorin, bei der Befragung nur diejenigen anzusprechen, die zu der heterogenen Gruppe von Unternehmen zählen. Somit konnte eine Vielzahl von potenziellen Unternehmen erreicht werden, die in bisherigen Studien keine Berücksichtigung fanden.

\section{Darstellung ausgewählter Ergebnisse der empirischen Studie}

\subsection{Branchenzugehörigkeit}

Die statistische Auswertung der Unternehmensdaten zeigte, dass eine marginale Mehrheit der Unternehmen zu der Maschinen/Metall-Branche (34,8 \%) gehört. Die Chemie-/ Kunststoff- und Pharma-Industrie vertrat ihren Branchenzweig mit 30,4\%, gefolgt von der Elektrotechnik-/Elektronik-Branche und „Anderen“ (Dienstleister, Logistik, Handel und Anlagebau) mit zu je 17,4\%.

\subsection{Durchführung von Vorbereitungsprogrammen/ Workshops für den Einsatz in der VR China}

Auf die Frage, ob in ihrem Unternehmen ein Vorbereitungsprogramm und/oder Workshop für den Einsatz in die VR China angeboten wird, antworteten nur 30,4\% der Probanden mit ja. Das heißt, mehr als zwei Drittel $(69,6 \%)$ werden ohne vorbereitende Maßnahmen für fast fünf Jahre nach China entsendet (die durchschnittliche Einsatzdauer der Befragten liegt bei 4,8 Jahren). Jene Aussagen spiegeln die bereits 1990 von Domsch/Lichtenberger und 1999 von Sader durchgefuihrten Studienergebnisse wieder. Diese bestätigten, dass nur ein relativ geringer Teil der zu Entsendenden Vorbereitungsmaßnahmen erhielten. ${ }^{1}$ So ermittelte Frau Sader in ihrer Arbeit, dass nur $24 \%$ aller Befragten ein Vorbereitungsseminar angeboten bekamen. ${ }^{2}$ Daher ist es erschreckend feststellen zu müssen, dass sich innerhalb von 12 Jahren kaum etwas in den Unternehmen bezüglich der Vorbereitungsmaßnahmen für einen Auslandseinsatz geändert hat.

In diesem Zusammenhang überrascht sehr, dass es sich bei den nicht vorbereiteten Probanden mit einem Anteil von 62,5\% vornehmlich um Mitarbeiter von Großunternehmen handelte. Im Gegensatz dazu zählten die vorbereiteten Unternehmen zu je 42,9\% sowohl zu den Kleinals auch zu den Großunternehmen. Dieses ist insoweit überraschend, da die Annahme bestand, dass Kleinunternehmen sich kein Vorbereitungsprogramm leisten kön-

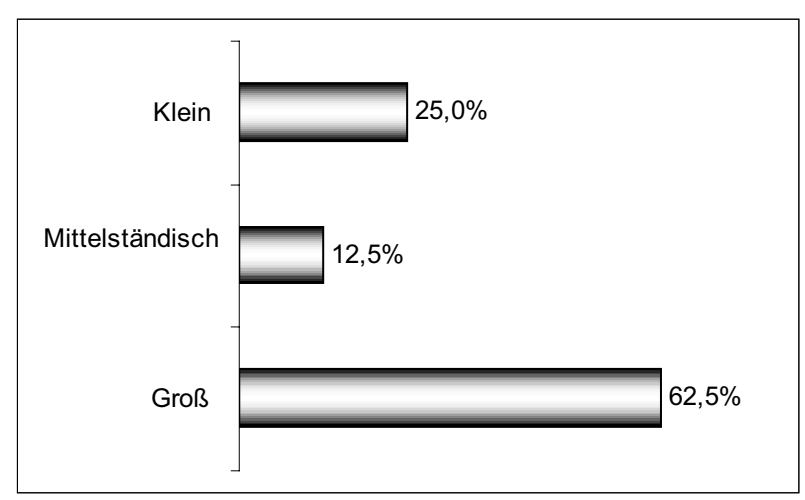

Abb.1: Angehörigkeit der nicht vorbereiteten Probanden

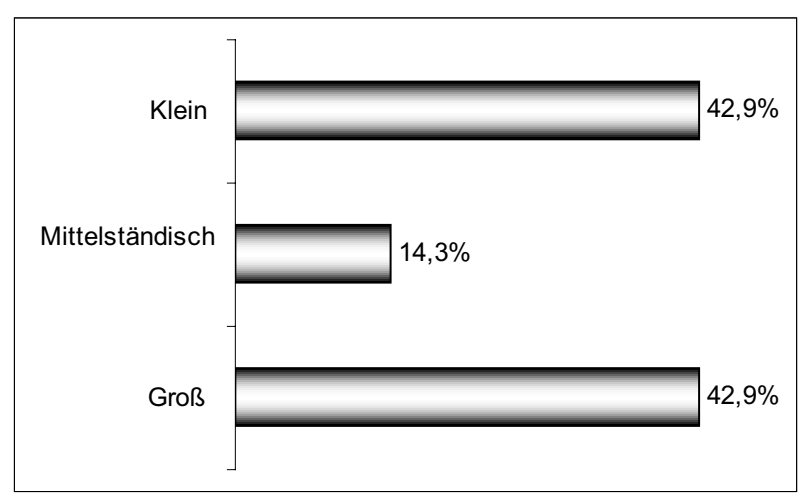

Abb. 2: Angehörigkeit der vorbereiteten Probanden

nen. Daher ist zu vermuten, dass die Kosten des entsprechenden Vorbereitungsprogramms nicht ausschlaggebend für das interne Vorbereitungsangebot sind, wenn sich selbst Kleinunternehmen solch ein Seminar leisten.

Die Bedeutung dieser Vorbereitungsmaßnahmen wird durch die folgende Tatsache noch deutlicher. So liegt der durchschnittliche Anteil der deutschen Belegschaft innerhalb eines Unternehmens in China bei 6,7\% und der der deutschen Führungskräfte bei 48,5\% (arithmetisches Mittel). Das heißt, obwohl die deutschen Mitarbeiter an der Gesamtbeschäftigtenzahl innerhalb des Unternehmens nur einen Anteil von 1/10 ausmachen, stellen sie trotz alledem knapp die Hälfte der Führungskräfte. Daraus resultiert folgende Berechnung: Wenn fast $70 \%$ der Probanden nicht durch ein chinaspezifisches Seminar vorbereitet wurden, sind somit 33,9\% der deutschen Führungskräfte vor Ort unvorbereitet. Folglich wurden mehr als zwei Drittel der Führungskräfte nicht speziell auf ihren Einsatz geschult. Dabei bleiben die vorbereiteten Kollegen im Durchschnitt 4,3 Jahre und die nicht Vorbereiteten im Durchschnitt 4,95 Jahre in der VR China. Damit ist ihre Einsatzdauer um fast sieben Monate länger. Diese Daten verdeutlichen, dass für längerfristige Einsätze in der VR China eine Vorbereitung auf kulturelle Unterschiede unumgänglich erscheint.

\subsection{Begründung für die Nichteinführung von Vorbereitungsprogrammen}

Als Erklärung für das Nichteinfuihren von Vorbereitungsprogrammen und/oder Workshops wurden folgende Gründe von den Nichtvorbereiteten genannt, warum solch ein Seminar in ihrem Unternehmen als nicht zweckmäßig erscheint. Hierbei ist alarmierenderweise 


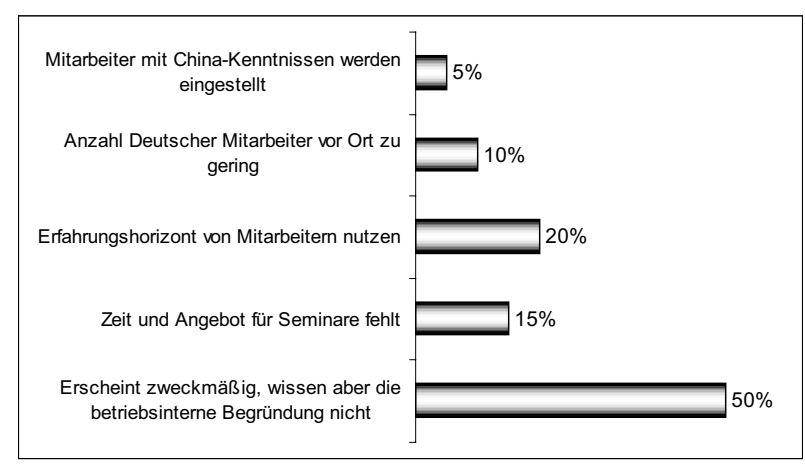

Abb. 3: Begründung für die Nichteinführung von Vorbereitungsprogrammen

festzustellen, dass $50 \%$ der Befragten die interne Begründung für die Nichtdurchfuihrung von Vorbereitungsmaßnahmen nicht kennen. Jedoch halten sie diese fuir einen erfolgreichen Aufenthalt in der VR China für sehr wichtig bzw. zweckmäßig.

$20 \%$ der Probanden gaben an, dass sie betriebsintern die gesammelten Erfahrungen der nach China entsandten Mitarbeiter nutzen. Dieses erscheint als problematisch, da sich die Kollegen auf die Erfahrungen und Angaben anderer verlassen. Viele dieser Erfahrungen beruhen auf subjektiven Empfindungen und Eindrücken, sodass aufgrund des fehlenden kulturellen Hintergrundwissens kein wirkliches kulturspezifisches Verständnis aufgebaut werden kann, um eine produktive interkulturelle Kommunikation zu fuihren und zu fördern. Dieses bedeutet nicht, dass niemand zu jenem in der Lage wäre. Es wird engagierte und motivierte Mitarbeiter geben, die willens sind, eigeninitiativ die Hintergründe für das Handeln und Denken der Chinesen zu ergründen und damit sehr kompetente Informationslieferanten darstellen. Jedoch kann durch die Angaben der Befragten angenommen werden, dass durch Zeitmangel sowohl in Deutschland (15\%) als auch in China (24 h-Dienst) die Möglichkeit fehlt, sich mit den chinesischen Gegebenheiten auseinander zu setzen und vertraut zu machen.

In Anbetracht dessen, dass deutsche Arbeitnehmer knapp die Hälfte der Führungskräfte an der Gesamtführungskräftezahl in China darstellen, erscheint die Äußerung von zehn Prozent der deutschen Befragungsteilnehmer als nicht tragbar. Sie berichteten, dass die Anzahl der deutschen Beschäftigten in der VR China zu geringfuigig sei, als dass sich ein Vorbereitungsprogramm lohnen würde. Dennoch ergab die Datenauswertung, dass 43,5\% der Probanden vor ihrer Entsendung gezielt auf die chinesische Geschichte, Kultur und die Mentalität vorbereitet wurden. Die verbleibenden Befragungsteilnehmer (56,5\%) erhielten keinen speziellen Unterricht in den genannten Aspekten. Dies lag in der Eigenverantwortung der Mitarbeiter.

\subsection{Beurteilung der betriebsinternen Vorbereitungsprogramme}

Insgesamt beurteilten die Probanden die betriebsinternen Vorbereitungsprogramme hinsichtlich Arbeitsplatzspezifik und Praxisorientierung in einem sehr ausgewogenen Verhältnis. So waren diese ihrer Meinung nach mit jeweils 28,6\% sowohl „zutreffend“, „,kaum zutreffend“ als auch „nicht zutreffend“, 14,3\% hielten sie fuir „sehr zutreffend“. Dies zeigt, dass 57,2\% der betrieblichen Seminare zu wenig auf Praxis- und spezifische Arbeitsplatzinformationen der Mitarbeiter eingehen. Ein gewisses Maß an Theorie und Allgemeinwissen sollte vermittelt werden, es widerspricht sich jedoch nicht, dabei die jeweilige Branche und Arbeit mit einzubeziehen und so das Seminar speziell am Kunden auszurichten.

\subsection{Problem- und Konfliktentstehung im Umgang mit chinesischen Mitarbeitern}

Die Mehrheit der Befragungsteilnehmer (84,3\%) nannten, als Klassifizierungsmerkmal zusammengefasst, kulturelle Grundvoraussetzungen als Hauptursachen fuir Verständigungsschwierigkeiten im Umgang mit den chinesischen Mitarbeitern. Unter dieses Merkmal fiel die chinesische Sprache, Kultur, Denkweise und Wertvorstellungen. Sie würden erst die Probleme und Konflikte in der interkulturellen Kommunikation entstehen lassen.

Durch individuelle Bedingungen wie fehlende Zeit und mangelnde Kommunikation werden nach Auffassung der deutschen Führungskräfte seitens der Deutschen die zweithäufigsten Konfliktpotenziale $(9,8 \%)$ verursacht. Darüber hinaus tragen die Unkenntnis über das Vorgehen bei Problemen und von Bearbeitungsschritten zu keiner Behebung der Verständigungsschwierigkeiten bei.

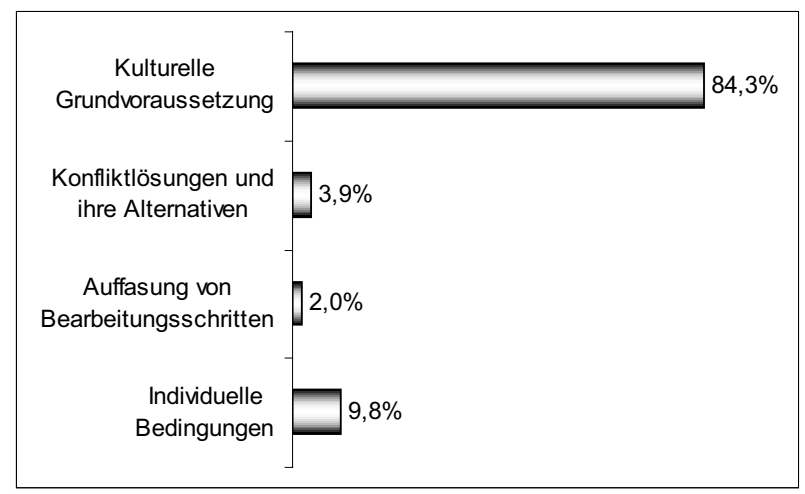

Abb. 4: Entstehung von Verständigungsschwierigkeiten

Da die Sprache das Denken, Fühlen und Wollen der Menschen eines Kulturkreises widerspiegelt, kann das Erlernen des Aufbaus bzw. des Konstruktes der chinesischen Sprache helfen, ein besseres Verständnis diesbezuiglich aufzubauen. Denn die Kenntnis des Sprachsystems hilft Verständigungsschwierigkeiten vorzubeugen. So wird gewährleistet, dass die deutschen Führungskräfte für den chinesischen Partner sensibilisiert werden.

In diesem Zusammenhang nannten mehr als die Hälfte $(52,2 \%)$ der Probanden, dass sie durch die Divergenzen in den Standards während ihrer Arbeit mit den chinesischen Mitarbeitern konfrontiert werden. Zu diesen Standards zählten u. a. mangelnde Ausbildung, unterschiedliche Identifikation und Auffassung der Unternehmensziele und Situationen, Hierarchieverständnis sowie Denkund Verhaltensmuster. 23,9\% der Befragten fuihrten aus, dass die fehlende Flexibilität (Mitdenken, eigenverant- 
wortliches Arbeiten, Kontrolle, Bereitschaft zu Problemvorschlägen) seitens der chinesischen Mitarbeiter zu Konflikten beitrage.

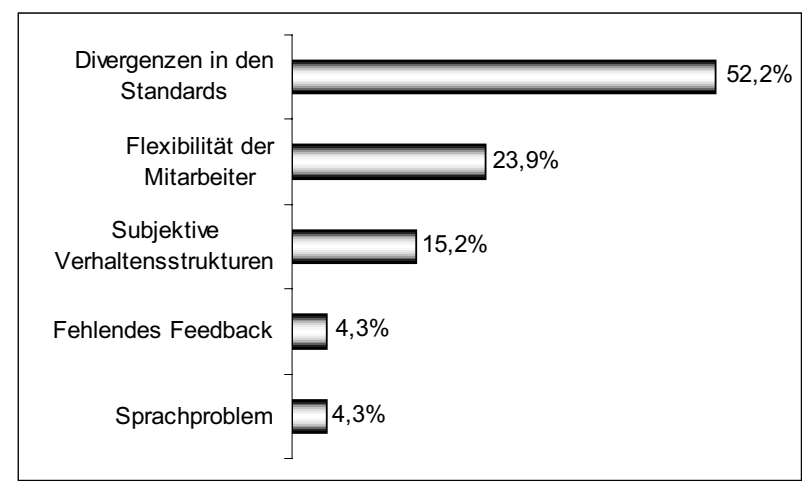

Abb. 5: Problementstehung durch Konfrontationen

Aufgrund ihrer kulturellen Prägung sind Chinesen es nicht gewohnt, in dem Maße eigenständig zu arbeiten, wie es die Erziehung in Deutschland von einem jeden Individuum verlangt. Daher dürfen die befragten Führungskräfte eigentlich nicht von ihren kulturellen Maßstäben auf die der Chinesen schließen. Da dies dennoch geschieht, wird mehr als deutlich, dass sie diesbezuiglich nicht ausreichend geschult worden waren. Das heißt, die Aussage von 43,5\% der Probanden, dass sie gezielt auf die chinesische Geschichte, Kultur und Mentalität vorbereitet worden seien, trifft nicht in dem Maße zu. Erschreckend sind daher die Angaben der Befragten, die bei den häufigen Verständigungsschwierigkeiten genannt wurden. So waren 84,3 \% auf kulturelle Grundvoraussetzungen zurückzuführen, wobei allein die fehlende Sprachkenntnis einen Anteil von 34,9\% ausmachte. Das heißt, fehlende Sprachkenntnisse sind zu einem erheblichen Teil fuir Probleme und Konflikte in der Zusammenarbeit mit den chinesischen Mitarbeitern verantwortlich.

\subsection{Sprachkenntnis}

Wie gerade aufgezeigt, ist die Sprachbarriere hauptverantwortlich für die Konfliktentstehung bei der deutsch-chinesischen Zusammenarbeit. Daher erstaunt auch nicht die Angabe auf die Frage, wie wichtig die Beherrschung der chinesischen Sprache im Chinageschäft sei. So erachteten $40 \%$ der Probanden jene als gar „nicht wichtig“. Nur 22,9\% der Befragungsteilnehmer bestätigen, dass Sprachkenntnisse von Vorteil wären, sich diese jedoch aufgrund der Komplexität der chinesischen Sprache und des Zeitmangels nicht bewerkstelligen ließen. Einige der Befragungsteilnehmer (14,3\%) antworteten sogar, dass sie die chinesische Sprache gar nicht zu lernen hätten, da Englisch sowieso die Unternehmenssprache sei. Ebenfalls gaben in diesem Tenor 14,3\% der Führungskräfte an, in die sprachliche Aus- und Weiterbildung der chinesischen Mitarbeiter zu investieren. Jene sollten erst einmal besser Englisch lernen. Diese sehr arrogante und ignorante Einstellung zeigt jedoch sehr vehement, dass $68,6 \%$ der deutschen Führungskräfte als Gast in der VR China verlangen, dass die chinesischen Mitarbeiter ihre Englischfähigkeiten verbessern müssen, bevor die Führungskräfte selber in Betracht ziehen, die chinesische Sprache zu erlernen.

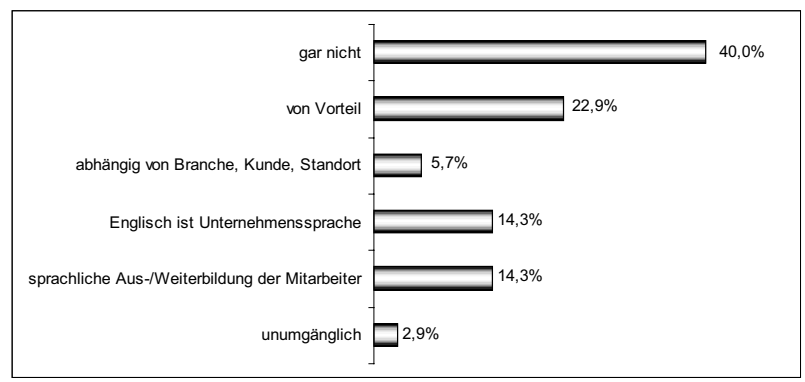

Abb. 6: Beherrschung der chinesischen Sprache

Nur 2,9\% der befragten Teilnehmer sind der Meinung gewesen, dass die Beherrschung der chinesischen Sprache unumgänglich sei.

Dabei sollten die Befragungsteilnehmer es sich nicht zu einfach machen, denn die Beherrschung einer Fremdsprache ist nicht gleichbedeutend mit der Abschaffung von Verständnisschwierigkeiten. Dieses ist nicht der Fall, da innerhalb einer jeden Fremdsprache die jeweiligen kulturspezifischen Varietäten und Kontextdualisierungshinweise der Muttersprache weiterhin beibehalten und angewandt werden. D. h. sowohl der Deutsche als auch der Chinese werden miteinander in Englisch kommunizieren und dennoch Verständnisprobleme nicht aus dem Weg gehen können. Infolgedessen sind Englischkenntnisse keine Garantie für eine fehlerfreie gegenseitige Verständigung, weil die unterschiedlichen Konventionen, Einstellungen, Werte, Normen und Verhaltensweisen nicht in dem Moment aufgegeben werden, in dem eine andere als die Muttersprache gesprochen wird.

Daher plädiert die Autorin, obwohl er sich der Schwierigkeit des Erlernens der chinesischen Sprache bewusst ist, dafür, Grundkenntnisse dieser Sprache zu erwerben, um zum einen Respekt gegenuiber den chinesischen Mitmenschen zeigen und zum anderen die Logik, Denk- und Verhaltensweise besser verstehen zu können.

\subsection{Primäres Ziel eines Vorbereitungstrainings}

Was die Probanden zu Anfang als Verständigungsschwierigkeiten bzw. Konfliktursachen definierten, spiegelte sich auch tendenziell in den wichtigsten Vorbereitungszielen eines möglichen Seminars wieder, um einen wirkungsvollen Chinaeinsatz zu ermöglichen und zu gewährleisten. Daher kann behauptet werden, dass insgesamt die Führungskräfte sehr wohl zum einen einschätzen können und zum anderen eine eindeutige Vorstellung haben, was für sie für einen erfolgreichen Aufenthalt von Bedeutung ist, trotz mangelnder Vorbereitung.

In den Augen der Probanden zählten aufgrund dessen folgende fünf Themenkomplexe zu den wichtigsten Zielen eines Vorbereitungstrainings:

1. vertraut sein mit dem Gastland und deren Kultur $(15,7 \%)$

2. auf interkulturelle Konflikte vorbereitet sein $(14,8 \%)$,

3. Kenntnisse über die kulturspezifische Sensibilität $(13,9 \%)$

4. Aufbau von kultureller Toleranz (12,25\%) sowie

5. kultureller Flexibilität $(10,4 \%)$. 
Leider kann diese sehr genaue Vorstellung der deutschen Führungskräfte nicht auf die der Arbeitgeber übertragen werden. So legen die Verantwortlichen bei der Personalauswahl gesteigerten Wert auf die überdurchschnittlichen Fachqualifikationen der in Betracht kommenden Führungskräfte. Dabei sind für die Befragungsteilnehmer die „Soziale Kompetenz“, das heißt Persönlichkeitsmerkmale, viel prägnanter für die Auswahl.

Dieses verdeutlicht die divergenten Vorstellungen der beteiligten Parteien uiber die Situation in der VR China. So existiert ein unterschiedliches Bild von den herrschenden Gegebenheiten vor Ort in den Köpfen der Vorgesetzten und Arbeitnehmer. Das bedeutet wiederum, dass kein informeller Austausch zwischen diesen beiden Gruppen stattfindet.

\section{Quintessenz}

Die Annahme, dass die zu entsendenden deutschen Führungskräfte nicht ausreichend vorbereitet werden, hat sich anhand der Studie und der Ergebnisse bestätigt. Somit ist es für die Vielzahl der befragten Unternehmen eine Ausnahme und nicht die Regel, interkulturelle Vorbereitungsprogramme und/oder Workshops für den Chinaeinsatz durchzufuihren.

Das veranschaulicht, dass sowohl interkulturelle Vorbereitungen als auch das (chinaspezifische) interkulturelle Management-Training bis heute keinen hohen Stellenwert innerhalb der befragten Unternehmen einnehmen und sich damit im Anfangsstadium befinden, obwohl diese von der Mehrheit der befragten Führungskräfte gefordert bzw. als wünschenswert erachtet werden.

Erschreckend ist allerdings die Tatsache, dass seit den Studien von M. Domsch und B. Lichtenberger im Jahr 1990 die Studienergebnisse bezüglich des Angebots von Vorbereitungsprogrammen und/oder Workshops keinen erwähnenswerten Veränderungen unterlagen. Vielmehr liegen die Ergebnisse dieser wissenschaftlichen Arbeit auf gleicher Ebene mit den Ergebnissen der Arbeit vor 12 Jahren.

a. Dabei ist die interkulturelle Vorbereitung wichtiger als je zuvor, denn die VR China hat sich nach Jahrhunderten der Unterdrückung, Fremdherrschaft und innerer Zerrissenheit zu einer neuen aufkommenden Wirtschaftsgröße entwickelt. Unter Führung der Kommunistischen Partei Chinas (KPCh) spielt das Land aufgrund seiner politischen und militärischen Stärke eine immer bedeutsamer werdende internationale Rolle. So ist China auf der internationalen politischen Bühne zu einem wichtigen Akteur in der Weltpolitik und -wirtschaft geworden (z. B. Sitz im Ständigen UN-Sicherheitsrat-Ausschuss, damit Veto-Recht).

Mit einer Fläche von 9,562 Mio. qkm und einer Einwohnerzahl von 1,26 Mrd. ${ }^{3}$ Menschen stellt die VR China ein Fünftel der Weltbevölkerung dar. Damit gilt China bei ausländischen Unternehmen als der größte und geschlossene und zu erschließende Markt der Erde, der ein hohes Potenzial an zukünftigen Konsumenten generiert. Dementsprechend müssen sich langfristig die Strategien deutscher Konzerne an diesem wichtigen Zukunftsmarkt orientieren.

Festzustellen ist jedoch, dass die Rangstellung Deutschlands, die sie innerhalb der Weltwirtschaft an der Volkswirtschaft Chinas einnimmt, interessanterweise weit unter seinen wirtschaftlichen Möglichkeiten liegt. Zwar werden immer die Paradebeispiele der deutschen Wirtschaft wie Volkswagen, Hoechst, BASF, Bayer, Henkel, Siemens, Thyssen Krupp, Dresdner Bank und Allianz ${ }^{4}$ in diesem Zusammenhang genannt, aber die Engagements dieser Unternehmen können nicht über die tatsächliche wirtschaftliche Situation hinwegtäuschen.

Es ist richtig, dass Deutschland knapp fünf Prozent des Handelsvolumen trägt, jedoch nur drei Prozent der Direktinvestitionen. ${ }^{5}$ „Für die viertgrößte Volkswirtschaft der Welt ... eine sehr magere Bilanz."6 Noch deutlicher drücken die Jointventure- Abschlüsse ausländischer Firmen die Tendenz Deutschlands aus. So wurden über 300.000 Jointventure-Verträge mit China abgeschlossen, wovon die BRD nur einen Anteil von 0,25 Prozent trägt. 7

Um dieses Manko zu beheben, beabsichtigen der deutsche Bundeskanzler Gerhard Schröder und Außenminister Joschka Fischer die Beziehung zu China weiter auszubauen und zu fördern.

Ausgehend von den aufgezeigten Daten, erscheint es zwingend notwendig, dass die deutschen Führungskräfte fuir ihren Auslandsaufenthalt vorbereitet und geschult werden, um

- einen optimalen Einsatz für das deutsche Unternehmen im Ausland,

- eine Umsatz- und Absatzmaximierung für die Unternehmen und

- eine Erhöhung des Marktanteils am chinesischen Markt für das betreffende deutsche Unternehmen zu ermöglichen.

b. Die durchgeführte wissenschaftliche Arbeit hat sehr deutlich gemacht, dass die interkulutrelle Vorbereitung Basis für ein erfolgreiches Engagement des Unternehmens in der VR China ist. Demzufolge muss als Voraussetzung fuir eine grundlegende Verbesserung der Situation deutscher Führungskräfte im Auslandsdienst die Teilnahme und Durchfuihrung eines Vorbereitungstrainings und/oder Workshops verpflichtend sein.

c. Wäre ein solches Seminar nicht bindend, dann ist keine Verbesserung der Situation der deutschen Führungskräfte vor Ort zu erwarten. Die dargestellte heutige unbefriedigende Situation würde sich also nicht verändern.

\section{d. Nur wer}

- den kulturspezifischen Herausforderungen gewachsen ist und diese kennt,

- gut und ausreichend für einen Chinaaufenthalt vorbereitet ist und dementsprechend in der Lage ist, alle 
wichtigen Faktoren zu berücksichtigen, der ist befähigt, die Grundbedingung fuir einen erfolgreichen Auslandseinsatz zu erfuillen.

\section{Anmerkungen}

1 Vgl. Domsch, M./Lichtenberger, B.: Vorbereitungsmaßnahmen für den Auslandseinsatz, 1990, S. $400 \mathrm{ff}$.

2 Vgl. Sader, K.: Deutsche Mitarbeiter in China, 1999, S. 143

3 Vgl. Maurer, J./Hüssen, H.-P.: Wirtschaftstrends, 2002, S. 7

4 Vgl. Eli, M.: China als Wirtschaftspartner und Konkurrent, 1997, S. $34 \mathrm{ff}$.

5 Vgl. Maurer, J./Hüssen, H.-P.: Wirtschaftstrends, 2002, S. 36, 42

6 Ederer, G./Franzen, J.: Der Sieg des himmlischen Kapitalismus, 1996, S. 384

7 Vgl. Ederer, G./Franzen, J.: Der Sieg des himmlischen Kapitalismus, 1996, S. 384

\section{Literaturhinweise}

Domsch, M./Lichtenberger, B.: Vorbereitungsmaßnahmen für den Auslandseinsatz, in: Betriebswirtschaftliche Forschung und Praxis, Herne, 42/1990

Ederer, Günter/Franzen, Jürgen: Der Sieg des himmlischen Kapitalismus - Wie der Aufstieg Chinas unsere Zukunft verändert, Verlag Moderne Industrie, Landsberg/Lech 1996

Eli, Max: China als Wirtschaftspartner und Konkurrent - Bericht über ein China-Symposium, das am 27. und 28 Mai 1997 im World Trade Center zu Dresden stattfand, ifo Institut, Dresden 1997

Maurer, Jürgen/Hüssen, Hans-Peter: VR China, Hongkong, SVR - Wirtschaftstrends - Zum Jahreswechsel 2001/02, Bundesagentur für Außenwirtschaft (bfai) Köln 2002

Sader, Karoline: Deutsche Mitarbeiter in China - Eine Analyse und Bewertung verschiedener Akkulturationsmuster, Mensch \& Buch Verlag, Berlin 1999

\section{Autoren}

Dipl.-Betriebswirtin (FH) Stephanie Nagel

Technische Fachhochschule Wildau

Fachbereich Betriebswirtschaft/Wirtschaftsinformatik

Dr. phil. Dirk Forschner

Technische Fachhochschule Wildau

China-Institut

Telefon +493375 508-365 und +493379 208740

E-Mail: dforsch@sprz.tfh-wildau.de 